\title{
Vecrotining Fascitis
}

\author{
K. Anbarasi, S. Sathasiva Subramanian, B. Shobana Devi \\ From the Department of Oral Medicine \& Radiology, Faculty of Dental Sciences, \\ Sri Ramachandra University, Porur, Chennai - 6001 16, Tamil Nadu, India.
}

\begin{abstract}
:
Necrotizing infections are characterized by tissue destructions, severe systemic toxicity and high mortality rate. Thrombosis of blood vessels and massive tissue loss facilitate abundant bacterial spread along the facial planes. Necrotizing fasciitis (NF) and myonecrosis are two rare entities of different depth of skin infections, which spreads at an alarming rate causing bacteraemia, organ failure, shock and death. Clinical indicators of necrotizing fasciitis include severe pain, rapidly spreading edema, bulla formation and toxic shock syndrome. Though NF is very rare, the clinician should not anticipate the chance of remote probability of occurrence, which may pose a lethal risk for the patients.
\end{abstract}

We present an unusual report of NF in a 35 years old female. Inspite of providing multidisciplinary intensive care therapy, this idiopathic, monomicrobial, acute form of NF turned fatal at the end of 36 hours.

Key words: Necrotizing Fasciitis, Klebsiellalnfections, Cellulitis, Bacteraemia, Edema.

\section{Introduction}

Joseph Jones first introduced necrotizing soft tissue infections (NSTI) in 1871 [1], which involves skin, subcutaneous tissue and muscles but does not cause myonecrosis and is potentially fatal. Necrotizing fasciitis is a rapidly spreading polymicrobial type of NSTI that affects primarily medically compromised patients and those with trauma or surgical wounds. Rarely its occurrence can be idiopathic in nature.

The infection spreads along the facial planes causing vascular thrombosis and ischemia. This facilitates angiothrombotic microbial invasion and liquefactive necrosis of superficial facia [2]. Once significant skin ischemia occurs, blisters or bulla formation takes place as a sign of ischemia-induced necrolysis and it is pathognomonic for NF [3]. Early diagnosis is often difficult as the infection starts at the plane of subcutaneous fat and deep facia with initial sparing of the epidermal and dermal layers [4]. Prognostic opportunity is enhanced if the first fasciotomy was carried out within $24 \mathrm{hrs}$ of necrotic manifestation. The present case is the sequel of misdiagnosis in the early golden hours and the unfavourable outcome even with extreme care in the later stage.

\section{Corresponding Author: Dr. K Anbarasi}

Email: anbarasi815@gmail.com

Received: September 14, 2013 | Accepted: October 15, 2013 | Published Online: November 10, 2013 This is an Open Access article distributed under the terms of the Creative Commons Attribution License (creativecommons.org/licenses/by/3.0)

Conflict of interest: None declared | Source of funding: Nil | DOl: http://dx.doi.org/10.17659/01.2013.0092 


\section{Case Report}

A 35-year-old female patient reported with multiple wounds on left side of the face since one week. We were informed through family members that, a month before she had undergone for extraction of left upper molar tooth and on the following week, she noticed erythematosus discoloration on the left facial skin and experienced severe pain. The patient reported to her physician and subjected for routine lab investigations $(\mathrm{Hb} \%$, plasma glucose level, $E S R$, total and differential leukocyte count) and the values were in normal range and was advised antibiotics, and analgesics. Within week duration, she developed multiple blisters on the same side that cracked with watery discharge, lead to large ulcers, and suffered with high fever. Again, she was advised to continue the medication along with topical antiseptic cream. As there was no relief, she was brought to our hospital. During examination, she found difficult to orient and co-operate. Her body temperature was $102^{\circ} \mathrm{F}$. Other noticeable vital findings include increased respiratory rate ( 25 per minute), heart rate, (130 beats per minute). Her BP was $60 / 90 \mathrm{mmHg}$. Left facial skin appeared dry and crusted with hard consistency on palpation. An area of approximately $2 \times 2.5 \mathrm{~cm}$ skin loss with underlying tissue exposure was evident adjacent to the left commeasures of lip and in the preauricular region, an intact bulla was evident [Fig.1]. On the temporal region, $2 \times 2 \mathrm{~cm}$ necrotic surface with fetid purulent discharge was noticed. The borders of these necrotic lesions were indistinct. Diffuse, soft and tender swelling was palpable on the left side of the head. Intra-orally, extracted socket revealed no abnormal findings.

We posted our patient for multidisciplinary approach in the intensive care unit and extended the investigations along with emergency management. Differential blood count showed leukocytosis $\left(21,010 / \mathrm{mm}^{3}\right)$ with abundant neutrophils $(91.2 \%)$. Biochemical investigation was remarkable for the

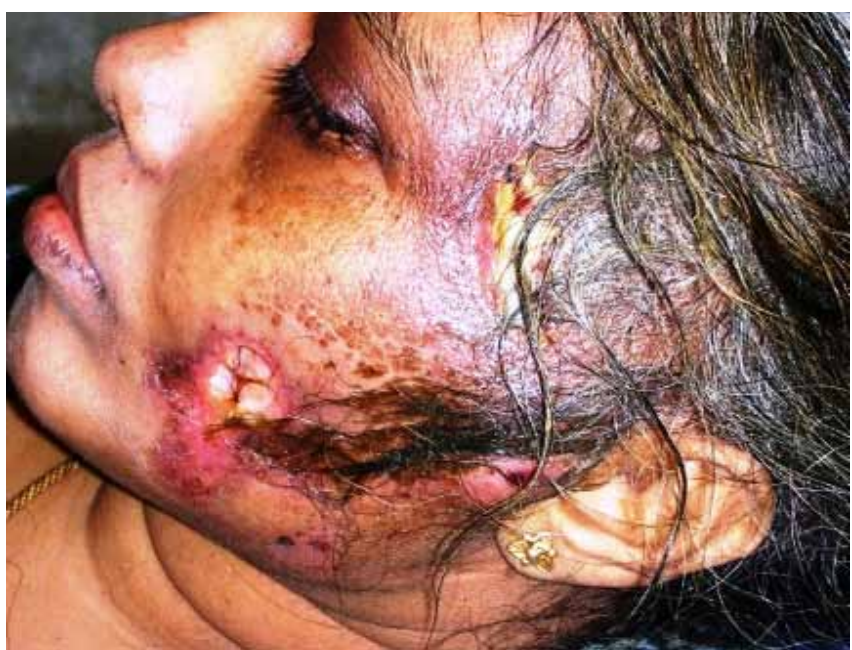

Fig.1: Profile view showing multiple necrotic ulcers, skin crustations and an intact bulla in the pre auricular region.

values of blood urea $(51 \mathrm{mg} / \mathrm{dL})$, creatinine $(2.4$ $\mathrm{mg} / \mathrm{dL})$, and $C$ - reactive protein $(180 \mathrm{mg} / \mathrm{L})$. Albumin level was lowered to $1.4 \mathrm{gm} / \mathrm{dL}$ and sodium level to $120 \mathrm{mmol} / \mathrm{L}$. Patient's random glucose value was $132 \mathrm{mg} / \mathrm{dL}$ and her hemoglobin level was $11 \mathrm{gm} / \mathrm{dL}$.

Extensive investigations were carried out to identify the causative organism through wound swab and blood culture techniques (BACTEC 9240). Klebsiella pneumoniae was isolated from the samples and subjected for antibiotic susceptibility using disc diffusion method which revealed susceptibility to Amikacin, Cefoperazone with sulbactum, Ciprofloxacin, Imipenem and Piperacillintazobactum. Correlating the clinical and laboratory data, we concluded craniofacial necrotizing fasciitis as diagnosis. We applied LRINEC [5] (Laboratory Risk Indicator for Necrotizing Fasciitis) scoring tool developed by Chin-Ho Wong et al., to substantiate our diagnosis and is strongly consistent with NF [Table 1].

Initial therapy included intravenous fluids, vasopressors, broad-spectrum antibiotics and 
Table 1: LRINEC (Laboratory Risk Indicator for Necrotizing Fasciitis) scoring for the present case.

\begin{tabular}{|c|c|c|}
\hline LRINEC Variable & Patients report & Score for our patient \\
\hline $\begin{array}{l}\text { C-Reactive protein } \mathrm{mg} / \mathrm{L} \\
<150=0 \\
>150=4\end{array}$ & $180 \mathrm{mg} / \mathrm{L}$ & 4 \\
\hline $\begin{array}{l}\text { Total WBC, per } \mathrm{mm}^{3} \\
>15=0 \\
\begin{array}{ll}15-25=1 \\
<11=2\end{array}\end{array}$ & $21,010 / \mathrm{mm}^{3}$ & 1 \\
\hline 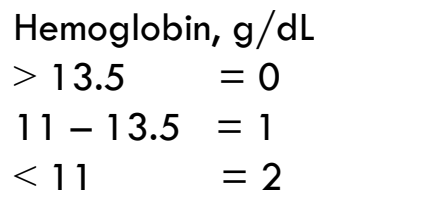 & $11 \mathrm{~g} / \mathrm{dL}$ & 1 \\
\hline $\begin{array}{l}\text { Sodium, } \mathrm{mmol} / \mathrm{L} \\
>135=0 \\
<135=2\end{array}$ & $120 \mathrm{mmol} / \mathrm{L}$ & 2 \\
\hline $\begin{array}{l}\text { Creatinine, } \mu \mathrm{mol} / \mathrm{L} \\
<141=0 \\
>141=2\end{array}$ & $212.16 \mu \mathrm{mol} / \mathrm{L}$ & 2 \\
\hline
\end{tabular}

ventilation support. After the antibiotic sensitivity report, specific IV antibiotic regimen was administered (Tazobactam sodium and Piperacillin sodium).

Wound debridement was carried out twice on the same day of admission. During the procedure, there was total lack of bleeding but foul smelling pus discharge was present. Necrotic tissues were excised despite the fact that the spread of necrosis was at an alarming rate. With no discernible changes, the patient expired after 36 hours of admission.

\section{Discussion}

Necrotizing fasciitis (NF) is a progressive, rapidly spreading infection in the deep fascia with secondary necrosis of the subcutaneous fat and fascia, which can be life threatening without prompt recognition, surgical intervention and antibiotic therapy [6]. Wilson in 1952 first coined this designation to explain the necrosis of fascia with relative sparing of the underlying muscles [7]. Polymicrobial infection (type I NF) accounts for $90 \%$ of cases where as monomicrobial infection (type II NF) occurs only in $10 \%$ cases [8]. The causative agents have classically been described as group A betahemolytic streptococci and staphylococci including obligate anaerobic bacteria [9]. The predisposing factors include diabetes, immunosuppressive drugs, malnutrition, renal and peripheral vascular diseases and intravenous drug abuse. Surgical and traumatic wounds contusion and varicella infections are also 
reported as initiating factors. $K$. pneumoniae is rarely accountable for the monomicrobial form of NF especially in immune compromised individuals and Wong et al. first reported such a case, in 1996 [10]. To the best of our knowledge, this is the first reported cases of $K$. pneumoniae induced NF involving craniofacial region in a young adult immunocompetent woman.

Spread of infective organism through the tissue planes results in necrosis of dermal vessels and subcutaneous tissue hypoxia. This enhances the growth of facultative anaerobes by reducing the function of polymorphs and encouraging anaerobic proliferation by decreasing oxidation/ reduction potential. Once the organism reaches the connective tissue, the infection spreads rapidly and is difficult to stop. Majority of these infections affects extremities (lower $28 \%$ and upper $27 \%$ ) followed by perineum $(21 \%)$ and trunk (18\%). Head and neck involvement is only $5 \%\left[\begin{array}{ll}1 & 1\end{array}\right]$.

No unique signs or symptoms give a warning to diagnose this fulminate condition in the early stage. Severe pain disproportionate to the lesion size may be the first striking feature. Pain may be followed by edema, indurations, red-blue discoloration of skin that changes to pathognomonic gray blue, illdefined patches at the end of 36 hours. Wooden hard consistency on palpation is characteristic at this stage [10]. Necrosis starts on the $5^{\text {th }}$ or $6^{\text {th }}$ day with superficial crustations. Pealing of crusts exposes the underlying structures with a purulent discharge. Bulla formation will be the last manifestation and is discouraging sign of progression [2]. Lymphadenopathy is unusual. High temperature, lethargy and disorientation are frequent accompaniments. The proposed explanation for systemic shock is binding of bacterial exotoxins to the MHC class II portion of antigen presenting cells. These exotoxins act as superantigens and stimulate host's immune response resulting in the production of cytokines like TNF-X, IL-1, IL-6, and IL-2, which contributes to tissue destruction, shock and organ failure [10].

Elevated values of leukocytes, BUN and serum creatinine are the positive reports in suspected patients. LRINEC scoring system is helpful to diagnose NF and differentiate it from the other soft tissue infections like cellulitis and erysipelas by using the routine biochemical lab reports. As the biochemical and hematologic changes develop during the early stages of NF, LRINEC scoring system is valuable to derive the diagnosis. A score of $\geq 6$ is an indicator to suspect NF whereas a score of $\geq 8$ robustly validate the diagnosis [5].

Appropriate antibiotics and repeated surgical debridement of all necrotic tissue will be the first line in management. Vasopressors should be included to correct the hypotension enhanced by bacterial toxins and infammatory mediators. Treatment modalities that need more clinical trial to establish the beneficial results include hyperbaric oxygen, intravenous immunoglobulin (IVIG), and bovine thymic extract.

\section{Conclusions}

NF greatly mimics facial cellulites and prompt early diagnosis is desirable for the welfare of the patient. The rarity of NF itself provides sparse knowledge about the clinical manifestations and treatment protocol. The confirmatory diagnosis requires clinical, microbiological and biological integration. The present case was probably misdiagnosed during the initial stage as a sequel of dental infection and the golden hours were missed out. This case adds a remark on literature that every unusual manifestation following any dental procedure should be evaluated with great care and expert team support is mandatory to avoid the later crisis. 


\section{References}

1. Jones J. Investigation upon the nature, causes, and treatment of hospital gangrene as prevailed in the Confederate armies 18611865. In: Surgical Memoirs of the War of the Rebellion. US Sanitary Commission, New York.

2. Wong $\mathrm{CH}$, Wang YS. The diagnosis of necrotizing fasciitis. Curr Opin Infect Dis. 2005;18:101106.

3. Nazir Z. Necrotizing fasciitis in neonates. Pediatr Surg Int. 2005; $21: 641$ - 644.

4. Kuo YL, Shieh SJ, Chiu HY, Lee JW. Necrotizing fasciitis caused by Vibrio vulnificus: epidemiology, clinical findings treatment and prevention. Eur J Clin Microbiol Infect Dis. 2007;26:785-792.

5. Chin-Ho Wong, Lay-Wai Khin, Kien-Seng Heng, Kok-Chai Tan, Cheng-Ooi Low. The LRINEC (Laboratory Risk Indicator for Necrotizing Fasciitis) Score: A Tool for Distinguishing Necrotizing Fasciitis from Other Soft Tissue Infections. Critical Care Medicine. 2004;32:1535-1541.
6. Harmonson JK, Tobar MY, Harkless LB. Necrotizing Fasciitis. Clin Paediatr Med Surg. 1996; 13:635-646.

7. Wilson B. Necrotizing fasciitis. Am J Surg. 1952;18:416-431.

8. Chapnick EK, Abter El. Necrotizing soft tissue infections. Infect Dis Clin North Am. $1996 ; 10: 835-855$.

9. Umeda $M$, Minamikawa $T$, Komatsubara $H$, Shibuya Y, Yokoo S, Komori T. Necrotizing fasciitis caused by dental infection: a retrospective analysis of 9 cases and a review of the literature. Oral Surg Oral Med OralPathol Oral Radiol Endod. 2003; 95:283-290.

10. Wong $\mathrm{CH}$, Kurup A, Wang YS, Heng KS, Tan KC. Four cases of necrotizing fasciitis caused by klebsiella species. Eur J Clin Microbial Infect Dis. 2004; 23:403-407.

11. Golger A, Goldsmith CH, Pennie RA, Bain JR. Mortality in patients with necrotizing fasciitis. Plast Reconstr Surg.2007;1 19:1803-1807. 